\title{
ON THE DIMENSION THEORY OF OVERRINGS OF AN INTEGRAL DOMAIN( $\left.{ }^{1}\right)$
}

\author{
BY \\ JIMMY T. ARNOLD
}

Let $D$ be an integral domain with identity which has quotient field $L$. If there exists a chain $P \subset P_{1} \subset \ldots \subset P_{n}$ of $n+1$ prime ideals of $D$, where $P_{n} \subset D$, but no such chain of $n+2$ prime ideals, then we say that $D$ has dimension $n$ and we write $\operatorname{dim} D=n$ [6]. In [6] and [7] Seidenberg has shown that if $\operatorname{dim} D=n$, and if $D$ is a Noetherian domain or a Prüfer domain, then $\operatorname{dim} D\left[X_{1}, \ldots, X_{m}\right]=n+m$, where $X_{1}, \ldots, X_{m}$ are indeterminates over $D$. In the special case in which $\operatorname{dim} D=1$ he has proved that the following statements are equivalent.

(1) $\operatorname{dim} D\left[X_{1}\right]=2$.

(2) $\operatorname{dim} D\left[X_{1}, \ldots, X_{m}\right]=m+1$ for any $m$.

More recently Gilmer has established the equivalence of the following properties for an $n$-dimensional domain $D$ [1].

(3) Every domain between $D$ and $L$ has dimension less than or equal to $n$.

(4) $\operatorname{dim} D\left[t_{1}, \ldots, t_{n}\right] \leqq n$ for $\left\{t_{1}, \ldots, t_{n}\right\} \subseteq L$.

For $n=1$ he further showed that (3) and (4) are equivalent to (1).

In this paper we consider domains $D$ having finite dimension $n$ and having the property that each domain between $D$ and its quotient field has dimension less than or equal to $\omega$ for some positive integer $\omega \geqq n$. For such a domain we obtain equivalent statements analogous to statements (1)-(4). The main results of this paper are contained in Theorems 2 and 5.

Throughout this paper $D$ will denote an integral domain with identity having quotient field $L$, and $X, X_{1}, \ldots, X_{m}$ will denote indeterminates over $D$. By an overring of $D$ we mean an integral domain $D^{\prime}$ such that $D \subseteq D^{\prime} \subseteq L$. By a valuation overring of $D$ we mean an overring of $D$ which is a valuation ring. Our notation will be that of Zariski-Samuel [8] with the one exception: $\subset$ denotes proper containment and $\subseteq$ denotes containment.

I. If $\operatorname{dim} D=n$ and $\omega \geqq n$, we wish to find necessary and sufficient conditions in order that each overring of $D$ have dimension less than or equal to $\omega$. One such set of conditions is given by the following theorem.

THEOREM 1. Suppose that $\operatorname{dim} D=n$. Then the following statements are equivalent.

(1) Each overring of $D$ has dimension less than or equal to $\omega$.

Received by the editors July 7, 1967 and, in revised form, April 22, 1968.

(1) This paper is a portion of the author's doctoral thesis, which was written under the direction of Professor Robert W. Gilmer at Florida State University. 
(2) Each valuation overring of $D$ has dimension less than or equal to $\omega$.

(3) For $\left\{t_{1}, \ldots, t_{\omega}\right\} \subseteq L$, dim $D\left[t_{1}, \ldots, t_{\omega}\right] \leqq \omega$.

Proof. Clearly (2) and (3) follow from (1). To show that (2) implies (1) we suppose that $D^{\prime}$ is an overring of $D$ such that $\operatorname{dim} D^{\prime}>\omega$. Then there exists a chain $(0) \subset P_{1}$ $\subset \ldots \subset P_{s} \subset D^{\prime}$ of prime ideals of $D^{\prime}$ such that $s>\omega$. By Theorem 11.9 of [5, p. 37] there exists a valuation overring $V$ of $D^{\prime}$ such that $V$ has prime ideals $Q_{1}, \ldots, Q_{s}$ which lie over $P_{1}, \ldots, P_{s}$ respectively. It follows that $V$ is a valuation overring of $D$ such that $\operatorname{dim} V>\omega$.

The proof that (3) implies (1) is given by Gilmer in [1] for the case $\omega=n$. The same method of proof is used here.

Suppose that there exists an overring $D^{\prime}$ of $D$ such that $\operatorname{dim} D^{\prime} \geqq \omega+1$. Let (0) $\subset P_{\omega+1} \subset \ldots \subset P_{1} \subset D^{\prime}$ be a chain of prime ideals of $D^{\prime}$ and let $t_{i} \in P_{i}-P_{i+1}$, $1 \leqq i \leqq \omega$. If $P_{i}^{\prime}=P_{i} \cap D\left[t_{1}, \ldots, t_{\omega}\right], 1 \leqq i \leqq \omega+1$, then $P_{i}^{\prime}$ is a prime ideal of $D\left[t_{1}, \ldots, t_{\omega}\right]$ and $t_{i} \in P_{i}^{\prime}-P_{i+1}^{\prime}, 1 \leqq i \leqq \omega$. Now let $r / s \in P_{\omega+1}$, where $r, s \in D-\{0\}$. Then $r=s(r / s)$ is an element of $P_{\omega+1} \cap D$ so that $r \in P_{\omega+1}^{\prime}$. Further, $1 \notin P_{1}^{\prime}$ since $1 \notin P_{1}$. Thus, $(0) \subset P_{\omega+1}^{\prime} \subset \ldots \subset P_{1}^{\prime} \subset D\left[t_{1}, \ldots, t_{\omega}\right]$ and $\operatorname{dim} D\left[t_{1}, \ldots, t_{\omega}\right] \geqq \omega+1$.

Theorem 1 leads us to the consideration of domains $D$ such that $\operatorname{dim} D\left[t_{1}, \ldots, t_{\omega}\right]$ $\leqq \omega$ for any subset $\left\{t_{1}, \ldots, t_{\omega}\right\}$ of $L$. More generally, for a fixed positive integer $m$, we wish to find necessary and sufficient conditions in order that $\operatorname{dim} D\left[t_{1}, \ldots, t_{m}\right]$ $\leqq \omega$, where $\left\{t_{1}, \ldots, t_{m}\right\}$ is any subset of $L$, and where $\omega \geqq \operatorname{dim} D$. Sufficient conditions are given by the following theorem.

TheOREM 2. Suppose that $\operatorname{dim} D\left[X_{1}, \ldots, X_{m}\right]=\omega+m$. Then $\omega \geqq \operatorname{dim} D$, and given $\left\{t_{1}, \ldots, t_{m}\right\} \subseteq L$, we have $\operatorname{dim} D\left[t_{1}, \ldots, t_{m}\right] \leqq \omega$.

If $P$ is a prime ideal of an integral domain $R$, we shall denote by $h(P)(d(P))$ the height (depth) of $P$ in $R$. Before proving Theorem 2, we require Lemma 1.

Lemma 1. Let $t_{1}, \ldots, t_{m}$ be elements of $L$ and let $\phi$ be the canonical D-homomorphism from $D\left[X_{1}, \ldots, X_{m}\right]$ onto $D\left[t_{1}, \ldots, t_{m}\right]$ such that $\phi\left(X_{i}\right)=t_{i}, 1 \leqq i \leqq m$. If $Q$ is the kernel of $\phi$, then $Q$ has height $m$ in $D\left[X_{1}, \ldots, X_{m}\right]$.

Proof. We have $Q \cap D=(0)$, for if $d \in D$, then $\phi(d)=d$. Thus, if $N=D-\{0\}$, $Q$ extends to a proper prime ideal of

$$
\left(D\left[X_{1}, \ldots, X_{m}\right]\right)_{N}=D_{N}\left[X_{1}, \ldots, X_{m}\right]=L\left[X_{1}, \ldots, X_{m}\right] .
$$

Further, if $h(Q)=s$ in $D\left[X_{1}, \ldots, X_{m}\right]$, the extension of $Q$ has height $s$ in

$$
L\left[X_{1}, \ldots, X_{m}\right] \text {. }
$$

However, $\operatorname{dim} L\left[X_{1}, \ldots, X_{m}\right]=m$ so that $s \leqq m$.

Let $Q_{i}=Q \cap D\left[X_{1}, \ldots, X_{i}\right]$ for $1 \leqq i \leqq m$. If $t_{i}=a_{i} / b_{i}, a_{i}, b_{i} \in D$, then $b_{i} X_{i}-a_{i} \in Q_{i}$. Consequently, $Q_{i} \neq(0)$ and for $1 \leqq i \leqq m-1, Q_{i}\left[X_{i+1}\right] \subset Q_{i+1}$, since $b_{i+1} X_{i+1}$ $-a_{i+1} \notin Q_{i}\left[X_{i+1}\right]$. It now follows that $(0) \subset Q_{1}\left[X_{2}, \ldots, X_{m}\right] \subset \ldots \subset Q_{m-1}\left[X_{m}\right.$ $\subset Q_{m}=Q$ so that $h(Q) \geqq m$. Thus equality holds and the lemma is proved. 
Proof of Theorem 2. Suppose that $\left\{t_{1}, \ldots, t_{m}\right\} \subseteq L$ and let $Q$ be the kernel of the $D$-homomorphism $\phi$ of $D\left[X_{1}, \ldots, X_{m}\right]$ onto $D\left[t_{1}, \ldots, t_{m}\right]$, where $\phi$ is such that $\phi\left(X_{i}\right)=t_{i}$ for each $i$. Further, let $(0) \subset Q_{1} \subset \ldots \subset Q_{k} \subset D\left[t_{1}, \ldots, t_{m}\right]$ be a chain of prime ideals of $D\left[t_{1}, \ldots, t_{m}\right]$. Then there exists a chain

$$
Q \subset P_{1} \subset \ldots \subset P_{k} \subset D\left[X_{1}, \ldots, X_{m}\right]
$$

of prime ideals of $D\left[X_{1}, \ldots, X_{m}\right]$ such that $\phi\left(P_{i}\right)=Q_{i}, 1 \leqq i \leqq k$. Since $Q$ has height $m, m+k \leqq \operatorname{dim} D\left[X_{1}, \ldots, X_{m}\right]=m+\omega$. Therefore, $k \leqq \omega$ as we wished to show.

Theorem 5 shows that the conditions given in Theorem 2 are also necessary in order that $\operatorname{dim} D\left[t_{1}, \ldots, t_{m}\right] \leqq \omega$ for $\left\{t_{1}, \ldots, t_{m}\right\} \subseteq L$. However, before proving Theorem 5 we need several other results.

Theorem 3. Suppose that $\operatorname{dim} D\left[t_{1}, \ldots, t_{m}\right] \leqq \omega$ for $\left\{t_{1}, \ldots, t_{m}\right\} \subseteq L$. If $J$ is an integral domain containing $D$ such that $J$ is integral over $D$, and if $F$ is the quotient field of $J$, then $\operatorname{dim} J\left[s_{1}, \ldots, s_{m}\right] \leqq \omega$ for $\left\{s_{1}, \ldots, s_{m}\right\} \subseteq F$.

In order to prove Theorem 3, we use the following lemma.

LeMma 2. Let $f(X)=f_{n} X^{n}+\cdots+f_{1} X+f_{0} \in D[X], f_{n} \neq 0$, and let $s$ be a root of $f(X)$ in an extension field of $L$. Then $s$ is integral over $D\left[1 / f_{n}\right]$ and $f_{n} s$ is integral over $D$.

Proof. Since $f(X) / f_{n} \in D\left[1 / f_{n}\right][X]$ and $f(s) / f_{n}=0$, it follows that $s$ is integral over $D\left[1 / f_{n}\right]$. Also

$$
0=f_{n}^{n-1} f(s)=\left(f_{n} s\right)^{n}+f_{n-1}\left(f_{n} s\right)^{n-1}+\cdots+f_{1} f_{n}^{n-2}\left(f_{n} s\right)+f_{0} f_{n}^{n-1}
$$

so that $f_{n} s$ is integral over $D$.

Proof of Theorem 3. $F$ is algebraic over $L$ since $J$ is integral over $D$. Therefore, if $s_{1}, \ldots, s_{m}$ are elements of $F$, there exists $f_{i}(X) \in D[X]-\{0\}$ such that $f_{i}\left(s_{i}\right)=0$, $1 \leqq i \leqq m$. It follows from Lemma 2 that if $d_{i}$ is the leading coefficient of $f_{i}(X)$, then $s_{i}$ is integral over $D\left[1 / d_{1}, \ldots, 1 / d_{m}\right]$ for each $i, 1 \leqq i \leqq m$. Hence, $J\left[s_{1}, \ldots, s_{m}\right]$ is integral over $D\left[1 / d_{1}, \ldots, 1 / d_{m}\right]$. Therefore, $\operatorname{dim} J\left[s_{1}, \ldots, s_{m}\right]=\operatorname{dim} D\left[1 / d_{1}, \ldots, 1 / d_{m}\right]$ by $\left[6\right.$, Theorem 5]. But $\operatorname{dim} D\left[1 / d_{1}, \ldots, 1 / d_{m}\right] \leqq \omega$ since $\left\{1 / d_{1}, \ldots, 1 / d_{m}\right\} \subseteq L$.

COROLlary 1. Suppose that $\operatorname{dim} D\left[t_{1}, \ldots, t_{m}\right] \leqq \omega$ for $\left\{t_{1}, \ldots, t_{m}\right\} \subseteq L$. Then if $\left\{s_{1}, \ldots, s_{m}\right\}$ is a set of elements algebraic over $D$, we have $\operatorname{dim} D\left[s_{1}, \ldots, s_{m}\right] \leqq \omega$.

Proof. Suppose $f_{i}(X) \in D[X]-\{0\}$ is such that $f_{i}\left(s_{i}\right)=0,1 \leqq i \leqq m$. From Lemma 2 it follows that if $d_{i}$ is the leading coefficient of $f_{i}(X)$, then $J=D\left[d_{1} s_{1}, \ldots, d_{m} s_{m}\right]$ is integral over $D$. Moreover, $\left\{s_{1}, \ldots, s_{m}\right\}$ is a subset of the quotient field of $J$. Therefore, since $J\left[s_{1}, \ldots, s_{m}\right]=D\left[s_{1}, \ldots, s_{m}\right]$, it follows from Theorem 3 that $\operatorname{dim} D\left[s_{1}, \ldots, s_{m}\right] \leqq \omega$.

THEOREM 4. If each overring of $D$ has dimension less than or equal to $\omega$, then $\operatorname{dim} D\left[X_{1}, \ldots, X_{m}\right] \leqq \omega+m$. 
Proof. Suppose that $\operatorname{dim} D\left[X_{1}, \ldots, X_{m}\right]=m+k, k \geqq 0$. It follows from Theorem 2 of [6] that $k \geqq \operatorname{dim} D$ so we have $D=L$ if $k=0$. Therefore, since the theorem is true for $D=L$, we assume that $k>0$. By Theorem 11.9 of [5, p. 37], there exists a valuation overring $W$ of $D\left[X_{1}, \ldots, X_{m}\right]$ such that $\operatorname{dim} W \geqq m+k$. If $V=W \cap L$, then $V$ is a valuation overring of $D$.

Suppose now that $\operatorname{dim} V=\mu$. Then by assumption $\mu \leqq \omega$, and by Theorem 4 of [7], if $Z_{1}, Z_{2}, \ldots, Z_{r}$ is any set of indeterminates over $V$, then $\operatorname{dim} V\left[Z_{1}, \ldots, Z_{r}\right]$ $=\mu+r$. In particular, if $Y_{1}, \ldots, Y_{\mu+m}$ are indeterminates over $V\left[X_{1}, \ldots, X_{m}\right]$, then $\operatorname{dim} V\left[X_{1}, \ldots, X_{m}\right]\left[Y_{1}, \ldots, Y_{\mu+m}\right]=2 \mu+2 m$. Therefore, by Theorem 2, if $\delta_{1}, \ldots, \delta_{\mu+m}$ are elements of $L\left[X_{1}, \ldots, X_{m}\right]$, the quotient field of $V\left[X_{1}, \ldots, X_{m}\right]$; then $\operatorname{dim} V\left[X_{1}, \ldots, X_{m}\right]\left[\delta_{1}, \ldots, \delta_{\mu+m}\right] \leqq \mu+m$. It then follows from Theorem 1 that every overring of $V\left[X_{1}, \ldots, X_{m}\right]$ has dimension less than or equal to $\mu+m$. But $W$ is an overring of $V\left[X_{1}, \ldots, X_{m}\right]$ so we have $m+k \leqq \operatorname{dim} W \leqq m+\mu$. Therefore, $k \leqq \mu \leqq \omega$ so that $\operatorname{dim} D\left[X_{1}, \ldots, X_{m}\right] \leqq \omega+m$ as we wished to show.

Lemma 3. Suppose that $\operatorname{dim} D\left[t_{1}, \ldots, t_{m}\right] \leqq \omega$ for $\left\{t_{1}, \ldots, t_{m}\right\} \subseteq L$, and let $P$ be a prime ideal of $D$ such that $h(P)=k$. If $F$ is the quotient field of $D / P$, then

$$
\operatorname{dim}(D / P)\left[s_{1}, \ldots, s_{m}\right] \leqq \omega-k \quad \text { for }\left\{s_{1}, \ldots, s_{m}\right\} \subseteq F .
$$

Proof. $F$ is isomorphic to $D_{P} / P D_{P}$, since $D_{P} / P D_{P} \cong(D / P)_{P / P}[8$, p. 227], and $D / P$ is isomorphic to $\left\{d+P D_{P} \mid d \in D\right\} \subseteq D_{P} / P D_{P}$. Thus suppose that $\left\{s_{1}, \ldots, s_{m}\right\} \subseteq$ $D_{P} / P D_{P}$-say $s_{i}=t_{i}+P D_{P}$, where $t_{i} \in D_{P}$, and let $D^{\prime}=D\left[t_{1}, \ldots, t_{m}\right]$.

If $(0) \subset P_{1} \subset \ldots \subset P_{k}=P$ is a chain of prime ideals of $D$, then $(0) \subset P_{1} D_{P} \subset \ldots$ $\subset P_{k} D_{P}=P D_{P} \subset D_{P}$ is a chain of prime ideals of $D_{P}$ such that $P_{i} D_{P} \cap D=P_{i}$, $1 \leqq i \leqq k$. Now $D \subseteq D^{\prime} \subseteq D_{P}$ so that if $P_{i}^{\prime}=P_{i} D_{P} \cap D^{\prime}$, then $P_{i}=P_{i}^{\prime} \cap D$. Therefore, (0) $\subset P_{1}^{\prime} \subset \cdots \subset P_{k}^{\prime}=P^{\prime} \subset D^{\prime}$ is a chain of prime ideals of $D^{\prime}$ and $h\left(P^{\prime}\right) \geqq k$.

It is easily seen that

$$
\begin{aligned}
D^{\prime} / P^{\prime} & \cong\left\{d^{\prime}+P D_{P} \mid d^{\prime} \in D^{\prime}\right\} \\
& =\left\{f\left(t_{1}, \ldots, t_{m}\right\}+P D_{P} \mid f\left(X_{1}, \ldots, X_{m}\right) \in D\left[X_{1}, \ldots, X_{m}\right]\right\} \\
& =\left\{\left(d_{0}+\sum d_{n_{1} \cdots n_{m}} t_{1}^{n_{1}} \cdots t_{m}^{n_{m}}\right)+P D_{P} \mid d_{i} \in D\right\} \\
& \cong\left\{d_{0}+P D_{P}+\sum\left(d_{n_{1}} \cdots n_{m}+P D_{P}\right)\left(t_{1}+P D_{P}\right)^{n_{1}} \cdots\left(t_{m}+P D_{P}\right)^{n_{m}} \mid d_{i} \in D\right\} \\
& \cong(D / P)\left[s_{1}, \ldots, s_{m}\right] .
\end{aligned}
$$

But by assumption $\operatorname{dim} D^{\prime} \leqq \omega$, and we have seen that $h\left(P^{\prime}\right) \geqq k$. Therefore, $\operatorname{dim} D^{\prime} / P^{\prime} \leqq \omega-k$; that is, $\operatorname{dim}(D / P)\left[s_{1}, \ldots, s_{m}\right] \leqq \omega-k$, and the proof of Lemma 3 is complete.

Lemma 4. Let $P$ be a prime ideal of $D, P \neq D$, and let $Q_{1} \subset Q_{2} \subset \cdots \subset Q_{s}$ be a chain of prime ideals of $D\left[X_{1}, \ldots, X_{m}\right]$ such that $Q_{i} \cap D=P$ for each $i, 1 \leqq i \leqq s$. Then $s \leqq m+1$ and there exists a chain $P\left[X_{1}, \ldots, X_{m}\right]=\Gamma_{1} \subset \ldots \subset \Gamma_{m+1}$ of prime ideals of $D\left[X_{1}, \ldots, X_{m}\right]$ such that $\Gamma_{i} \cap D=P$ for each $i$ and such that $\left\{Q_{1}, \ldots, Q_{s}\right\}$ $\subseteq\left\{\Gamma_{1}, \ldots, \Gamma_{m+1}\right\}$. 
Proof. If $P^{e}=P\left[X_{1}, \ldots, X_{m}\right]$, then $Q_{1} / P^{e} \subset \ldots \subset Q_{s} / P^{e}$ is a chain of prime ideals $D\left[X_{1}, \ldots, X_{m}\right] / P^{e}=(D / P)\left[X_{1}, \ldots, X_{m}\right]$ meeting $D / P$ in $(0)$. Thus, it suffices to prove Lemma 4 for the case in which $P=(0)$. But if $P=(0)$, then

$$
\left(D\left[X_{1}, \ldots, X_{m}\right]\right)_{D-P}=L\left[X_{1}, \ldots, X_{m}\right] .
$$

Lemma 4 now follows from the results in [9, p. 194].

Lemma 5. Let $D$ be a quasi-local domain with maximal ideal $M$. If $\operatorname{dim} D=$ $n \leqq \omega \leqq m$ and if $\operatorname{dim} D\left[X_{1}, \ldots, X_{m}\right] \geqq \omega+m+1$, then there exists $a$ chain of prime ideals of $D\left[X_{1}, \ldots, X_{m}\right]$ of the form $M\left[X_{1}, \ldots, X_{m}\right] \supset Q_{\omega} \supset \ldots \supset Q_{1} \supset(0)$, where either $Q_{1}=P\left[X_{1}, \ldots, X_{m}\right]$ for some prime ideal $P$ of $D$, or $Q_{1} \cap D=(0)$ but $Q_{1} \cap D\left[X_{1}\right] \neq(0)$.

For convenience we number the following remark since it will be used repeatedly in the proof of Lemma 5.

Remark 1. If $\operatorname{dim} D\left[X_{1}, \ldots, X_{m}\right] \geqq \omega+m+1$, then by Theorem 4 some overring of $D$ has dimension greater than or equal to $\omega+1$. Hence, by Theorem 1 , we have $\operatorname{dim} D\left[t_{1}, \ldots, t_{\omega}\right] \geqq \omega+1$ for some $\left\{t_{1}, \ldots, t_{\omega}\right\} \subseteq L$ so that, by Theorem 2 , $\operatorname{dim} D\left[X_{1}, \ldots, X_{\omega}\right] \geqq 2 \omega+1$.

Proof of Lemma 5. If the lemma were true in the special case in which $m=\omega$, there would exist a chain of prime ideals of $D\left[X_{1}, \ldots, X_{\omega}\right]$ of the form

$$
M\left[X_{1}, \ldots, X_{\omega}\right] \supset Q_{\omega} \supset \ldots \supset Q_{1} \supset(0),
$$

where either $Q_{1}=P\left[X_{1}, \ldots, X_{\omega}\right]$ for some prime ideal $P$ of $D$, or $Q_{1} \cap D=(0)$ but $Q_{1} \cap D\left[X_{1}\right] \neq(0)$.

Since $\omega \leqq m$,

$$
M\left[X_{1}, \ldots, X_{m}\right] \supset Q_{\omega}\left[X_{\omega+1}, \ldots, X_{m}\right] \supset \ldots \supset Q_{1}\left[X_{\omega+1}, \ldots, X_{m}\right] \supset(0)
$$

is a chain of prime ideals of $D\left[X_{1}, \ldots, X_{m}\right]$ having the desired form.

Therefore, it suffices to prove Lemma 5 for the special case in which $\omega=m$. The proof will be by induction on $n$, where $n=\operatorname{dim} D$.

We first consider the special case in which there exists a chain of prime ideals $D\left[X_{1}, \ldots, X_{m}\right] \supset Q_{2 m+1} \supset \ldots \supset Q_{1} \supset(0)$ such that if $Q_{i} \cap D \neq(0)$, then $Q_{i} \cap D=M$, $1 \leqq i \leqq 2 m+1$. Since this is the case when $n=1$ we will have the first step of an induction argument.

By taking $P=(0)$ in Lemma 4 , we see that $Q_{m+1} \cap D \neq(0)$ so that, by hypothesis, $Q_{m+1} \cap D=M$. Then $Q_{m+1} \supseteq M\left[X_{1}, \ldots, X_{m}\right]$, and it follows from Lemma 4 that $M\left[X_{1}, \ldots, X_{m}\right]$ has depth $m$ in $D\left[X_{1}, \ldots, X_{m}\right]$. But $d\left(Q_{m+1}\right) \geqq m$, so $Q_{m+1}$ $=M\left[X_{1}, \ldots, X_{m}\right]$.

Since $Q_{m+1} \supset Q_{m}$, our assumption implies that $Q_{m} \cap D=(0)$. However, by Lemma $4, Q_{m} \cap D\left[X_{1}\right] \neq(0)$-say $Q_{m} \cap D\left[X_{1}\right]=Q_{1}^{\prime}$. If $D^{\prime}=D\left[X_{1}\right]_{Q_{1}^{\prime}}$, then $D^{\prime} \supseteq L$ since $Q_{1}^{\prime} \cap D=(0)$, and every valuation overring of $D^{\prime}$ has dimension less than or equal to one $\left[9\right.$, p. 50]. Therefore, by Theorem $1, D^{\prime}$ is a one-dimensional domain such that every overring has dimension less than or equal to one. It then follows 
from Theorem 4 that $\operatorname{dim}\left(D^{\prime}\right)\left[X_{2}, \ldots, X_{m}\right]=m$. Let $\left(Q_{1}^{\prime}\right)^{e}=\left(Q_{1}^{\prime}\right) D^{\prime}$. By Lemma 4, $\left(Q_{1}^{\prime}\right)^{e}\left[X_{2}, \ldots, X_{m}\right]$ has depth $m-1$ in $\left(D^{\prime}\right)\left[X_{2}, \ldots, X_{m}\right]$, so it is minimal. Since $\left(D^{\prime}\right)\left[X_{2}, \ldots, X_{m}\right]$ is a quotient ring of $D\left[X_{1}, \ldots, X_{m}\right]$ with respect to the multiplicative system

$$
D\left[X_{1}\right]-Q_{1}^{\prime}, Q_{1}^{\prime \prime}=\left(Q_{1}^{\prime}\right)\left[X_{2}, \ldots, X_{m}\right]=\left(Q_{1}^{\prime}\right)^{e}\left[X_{2}, \ldots, X_{m}\right] \cap D\left[X_{1}, \ldots, X_{m}\right]
$$

is minimal in $D\left[X_{1}, \ldots, X_{m}\right]$.

Now $Q_{1}^{\prime \prime} \subseteq Q_{m}$ and $Q_{1}^{\prime \prime} \cap D\left[X_{1}\right]=Q_{1}^{\prime}=Q_{m} \cap D\left[X_{1}\right]$. If $Q \supset Q_{m}$, then $h(Q) \geqq m+1$, so by Lemma 4 we have $Q \cap D \neq(0)$. Hence $Q \cap D\left[X_{1}\right] \neq Q_{1}^{\prime}$ since $Q_{1}^{\prime} \cap D \stackrel{(=)}{=}$. Since $Q_{1}^{\prime \prime}$ is minimal there exists, by Lemma 4 , prime ideals $Q_{2}^{\prime \prime}, \ldots, Q_{m-1}^{\prime \prime}$ of $D\left[X_{1}, \ldots, X_{m}\right]$ such that $(0) \subset Q_{1}^{\prime \prime} \subset Q_{2}^{\prime \prime} \subset \ldots \subset Q_{m-1}^{\prime \prime} \subset Q_{m}$. Then $M\left[X_{1}, \ldots, X_{m}\right]$ $\supset Q_{m} \supset Q_{m-1}^{\prime \prime} \supset \ldots \supset Q_{2}^{\prime \prime} \supset Q_{1}^{\prime \prime} \supset(0)$ is the desired chain.

We now assume that the result is true for $n<k$ and that $\operatorname{dim} D=k$. If

$$
\operatorname{dim} D\left[X_{1}, \ldots, X_{m}\right] \geqq 2 m+1
$$

and if $D\left[X_{1}, \ldots, X_{m}\right] \supset Q_{2 m+1} \supset \cdots \supset Q_{1} \supset(0)$ is a chain of prime ideals of

$$
D\left[X_{1}, \ldots, X_{m}\right]
$$

then, from what we have just shown, we may assume that $(0) \subset Q_{i} \cap D \subset M$ for some $i, 1 \leqq i \leqq 2 m+1$. Thus we choose $\alpha, 1 \leqq \alpha \leqq 2 m+1$, such that $Q_{\alpha} \cap D \neq(0)$ but $Q_{\alpha-1} \cap D=(0)$. (We take $Q_{0}=(0)$.) Suppose that $Q_{\alpha} \cap D=P$ and suppose that $\operatorname{dim} D_{P}=\mu$. By assumption $P \subset M$ so that $\mu<k$. Let $(0) \subset P_{1} \subset \ldots \subset P_{\mu-1} \subset P$ be a chain, having length $\mu$, of prime ideals of $D$ which are contained in $P$. Let $\lambda$ be the maximal length of a proper chain of prime ideals of $D\left[X_{1}, \ldots, X_{m}\right]$ which is contained properly between $Q_{\alpha}$ and $P\left[X_{1}, \ldots, X_{m}\right]$ (let $\lambda=-1$ if $Q_{\alpha}=P\left[X_{1}, \ldots, X_{m}\right]$ ), and let $t=(2 m+1)-(\alpha-1)=2 m-\alpha+2$. Then $P_{1}\left[X_{1}, \ldots, X_{m}\right]$ has depth greater than or equal to $t+\lambda+\mu-1$ in $D\left[X_{1}, \ldots, X_{m}\right]$.

If $t+\lambda+\mu \geqq 2 m+1$, then $\operatorname{dim}\left(D / P_{1}\right)\left[X_{1}, \ldots, X_{m}\right] \geqq 2 m=(m-1)+m+1$ and $m-1 \geqq \operatorname{dim} D / P_{1}$ (since $m \geqq \operatorname{dim} D$ and $P_{1} \neq(0)$ ). Taking $\omega=m-1$, Remark 1 implies that $\operatorname{dim}\left(D / P_{1}\right)\left[X_{1}, \ldots, X_{m-1}\right] \geqq 2(m-1)+1$. But $\operatorname{dim} D / P_{1}<\operatorname{dim} D=k$, so by the induction hypothesis there is a chain of prime ideals of

$$
\left(D / P_{1}\right)\left[X_{1}, \ldots, X_{m-1}\right]
$$

of the form $\left(M / P_{1}\right)\left[X_{1}, \ldots, X_{m-1}\right] \supset Q_{m-1}^{\prime \prime} \supset \ldots \supset Q_{1}^{\prime \prime} \supset(0)$. If for $1 \leqq i \leqq m-1, Q_{i}^{\prime}$ is the unique prime ideal of $D\left[X_{1}, \ldots ; X_{m}\right]$ such that $Q_{i}^{\prime} \supseteq P_{1}\left[X_{1}, \ldots, X_{m-1}\right]$ and $\left(Q_{\mathfrak{i}}^{\prime}\right) / P_{1}\left[X_{1}, \ldots, X_{m-1}\right] \cong Q_{\mathfrak{i}}^{\prime \prime}$, then

$$
M\left[X_{1}, \ldots, X_{m-1}\right] \supset Q_{m-1}^{\prime} \supset \ldots \supset Q_{1}^{\prime} \supset P_{1}\left[X_{1}, \ldots, X_{m-1}\right] .
$$

Clearly, $M\left[X_{1}, \ldots, X_{m}\right] \supset\left(Q_{m-1}^{\prime}\right)\left[X_{m}\right] \supset \ldots \supset\left(Q_{1}^{\prime}\right)\left[X_{m}\right] \supset P_{1}\left[X_{1}, \ldots, X_{m}\right]$ is a chain of the desired form.

We now suppose that $t+\lambda+\mu<2 m+1$. We first show the existence of a chain $M\left[X_{1}, \quad, X_{m}\right] \supset Q_{\beta}^{\prime} \supset \ldots \supset Q_{1}^{\prime} \supset P\left[X_{1}, \ldots, X_{m}\right]$ of prime ideals of $D\left[X_{1}, \ldots, X_{m}\right]$ such that $\beta+m+1 \geq t+\lambda$. 
Thus, if $t+\lambda \leqq m+\operatorname{dim} D / P$, we take $\beta+1=\operatorname{dim} D / P$. Then there exists a chain $M \supset Q_{\beta} \supset \ldots \supset Q_{1} \supset P$ of prime ideals of $D$ and $M\left[X_{1}, \ldots, X_{m}\right] \supset Q_{\beta}\left[X_{1}, \ldots, X_{m}\right]$ $\supset \ldots \supset Q_{1}\left[X_{1}, \ldots, X_{m}\right] \supset P\left[X_{1}, \ldots, X_{m}\right]$ is the desired chain. If, on the other hand, $t+\lambda \geqq \operatorname{dim} D / P+m+1$, we take $\beta$ to be such that $t+\lambda=\beta+m+1$. Then $\beta \geqq \operatorname{dim}$ $D / P$ and by assumption $t+\lambda+\mu<2 m+1$, so that $\beta+m+1=t+\lambda<2 m+1$. Therefore we have $\beta<m$; that is, $\operatorname{dim} D / P<m$. But $\operatorname{dim}(D / P)\left[X_{1}, \ldots, X_{m}\right] \geqq t+\lambda=\beta$ $+m+1$, so it follows from $\operatorname{Remark} 1$ that $\operatorname{dim}(D / P)\left[X_{1}, \ldots, X_{\beta}\right] \geqq 2 \beta+1$. Since $\operatorname{dim} D / P<k$, the induction hypothesis is applicable. Hence, using the same method of proof given above for $D / P_{1}$, there exist prime ideals $Q_{\beta}^{\prime}, \ldots, Q_{1}^{\prime}$ of $D\left[X_{1}, \ldots, X_{m}\right]$ such that $M\left[X_{1}, \ldots, X_{m}\right] \supset Q_{\beta}^{\prime} \supset \ldots \supset Q_{1}^{\prime} \supset P\left[X_{1}, \ldots, X_{m}\right]$.

We now consider the domain $D_{P}$. Since $Q_{i} \cap D \subseteq P$ for $1 \leqq i \leqq \alpha$, if we set $Q_{i}^{e}=Q_{i} D_{P}\left[X_{1}, \ldots, X_{m}\right], 1 \leqq i \leqq \alpha, P_{i}^{e}=P_{i} D_{P}, 1 \leqq i \leqq \mu-1$, and $P^{e}=P D_{P}$, then we have $(0) \subset Q_{1}^{e} \subset \ldots \subset Q_{\alpha}^{e},(0) \subset\left(P_{1}^{e}\right)\left[X_{1}, \ldots, X_{m}\right] \subset \ldots \subset\left(P_{\mu-1}^{e}\right)\left[X_{1}, \ldots, X_{m}\right] \subset\left(P^{e}\right)$ $\cdot\left[X_{1}, \ldots, X_{m}\right] \subseteq Q_{\alpha}^{e}, Q_{i}^{e} \cap D_{P}=(0)$ for $1 \leqq i \leqq \alpha-1, Q_{\alpha}^{e} \cap D_{P}=P^{e}$, and $\lambda$ is the maximum length of a proper chain of prime ideals of $D_{P}\left[X_{1}, \ldots, X_{m}\right]$ contained properly between $Q_{\alpha}^{e}$ and $\left(P^{e}\right)\left[X_{1}, \ldots, X_{m}\right]\left(\lambda=-1\right.$ if $\left.Q_{\alpha}^{e}=P^{e}\left[X_{1}, \ldots, X_{m}\right]\right)$. By Lemma 4 there is a chain of prime ideals of $D_{P}\left[X_{1}, \ldots, X_{m}\right]$ of the form

$$
\left(P^{e}\right)\left[X_{1}, \ldots, X_{m}\right]=H_{m+1} \subset H_{m} \subset \ldots \subset H_{1}
$$

such that $H_{i} \cap D_{P}=P^{e}$ for each $i$, and $Q_{\alpha}^{e}=H_{s}$ for some $s, 1 \leqq s \leqq m+1$. Then (0) $\subset Q_{1}^{e} \subset \ldots \subset Q_{\alpha-1}^{e} \subset H_{s} \subset \ldots \subset H_{1} \subset D_{P}\left[X_{1}, \ldots, X_{m}\right]$ is a chain of prime ideals of $D_{P}\left[X_{1}, \ldots, X_{m}\right]$ so that $\operatorname{dim} D_{P}\left[X_{1}, \ldots, X_{m}\right] \geqq \alpha-1+s$. But by assumption $\mu+\lambda+t<2 m+1$. Hence, $\mu+\lambda<2 m+1-t=\alpha-1$ and we have $\mu+\lambda+s<\alpha-1+s$. By choice of the integer $\lambda$ and the ideals $H_{1}, \ldots, H_{s}=Q_{\alpha}^{e}$, it follows from Lemma 4 that $\lambda+s=m$. Consequently, $\mu+m<\alpha-1+s$. By Lemma 4, $\alpha-1 \leqq m$ (since $i \leqq \alpha-1$ implies $Q_{i} \cap D=(0)$ ), and $s \leqq m+1$ by choice. Then $\alpha-1+s \leqq 2 m+1$, so we may choose $\gamma \leqq m$ such that $\alpha-1+s=\gamma+m+1$. We now have $\mu+m<\alpha$ $-1+s=\gamma+m+1 \leqq \operatorname{dim} D_{P}\left[X_{1}, \ldots, X_{m}\right]$, from which it follows that $\mu \leqq \gamma \leqq m$ (we recall that $\mu=\operatorname{dim} D_{P}$ ). Remark 1 now implies that $\operatorname{dim} D_{P}\left[X_{1}, \ldots, X_{y}\right]$ $\geqq 2 \gamma+1$.

Since $P \subset M, \operatorname{dim} D_{P}<k$, so by the induction hypothesis there is a chain of prime ideals of $D_{P}\left[X_{1}, \ldots, X_{\gamma}\right]$ of the form $P^{e}\left[X_{1}, \ldots, X_{\gamma}\right] \supset \Gamma_{\gamma}^{\prime} \supset \ldots \supset \Gamma_{1}^{\prime} \supset(0)$, where either $\Gamma_{1}^{\prime}=P^{\prime}\left[X_{1}, \ldots, X_{\gamma}\right]$ for some prime ideal $P^{\prime}$ of $D_{P}$, or $\Gamma_{1}^{\prime} \cap D_{P}=(0)$ but $\Gamma_{1}^{\prime} \cap D_{P}\left[X_{1}\right] \neq(0)$. If we let $\Gamma_{i}=\Gamma_{i}^{\prime} \cap D\left[X_{1}, \ldots, X_{\gamma}\right], 1 \leqq i \leqq \gamma$, then

$$
P\left[X_{1}, \ldots, X_{\gamma}\right] \supset \Gamma_{\gamma} \supset \ldots \supset \Gamma_{1} \supset(0)
$$

is a chain of prime ideals of $D\left[X_{1}, \ldots, X_{\gamma}\right]$. Further, if $\Gamma_{1}^{\prime}=\left(P^{\prime}\right)\left[X_{1}, \ldots, X_{\gamma}\right]$, then $\Gamma_{1}=\left(P^{\prime \prime}\right)\left[X_{1}, \ldots, X_{\gamma}\right]$ where $P^{\prime \prime}=P^{\prime} \cap D$; or, if $\Gamma_{1}^{\prime} \cap D_{P}=(0)$ but $\Gamma_{1}^{\prime} \cap D\left[X_{1}\right] \neq(0)$, then $\Gamma_{1} \cap D=(0)$ but $\Gamma_{1} \cap D\left[X_{1}\right] \neq(0)$. We now show that

$$
\begin{aligned}
M\left[X_{1}, \ldots, X_{m}\right] & \supset Q_{\beta}^{\prime} \supset \ldots \supset Q_{1}^{\prime} \supset P\left[X_{1}, \ldots, X_{m}\right] \\
& \supset L_{\gamma}\left[X_{\gamma+1}, \ldots, X_{m}\right] \supset \ldots \supset \Gamma_{1}\left[X_{\gamma+1}, \ldots, X_{m}\right] \supset(0)
\end{aligned}
$$

is the desired chain of prime ideals. 
Certainly $\Gamma_{1}\left[X_{\gamma+1}, \ldots, X_{m}\right]$ has the desired form, so it suffices to show that $\beta+\gamma+1 \geqq m$. But $\gamma+\lambda+s+1=\gamma+m+1=\alpha-1+s=2 m+1-t+s$. Hence, $\gamma+\lambda+1$ $=2 m+1-t$ so that $\gamma+\lambda+t+1=2 m+1$. By choice of $\beta, \beta+m+1 \geqq t+\lambda$ and it follows that $\gamma+\beta+m+2 \geqq \gamma+t+\lambda+1=2 m+1$. Therefore, $\beta+\gamma+1 \geqq m$ as we wished to show.

This completes the proof of Lemma 5.

We are now in position to show that the conditions given in Theorem 2 are necessary in order that $\operatorname{dim} D\left[t_{1}, \ldots, t_{m}\right] \leqq \omega$ for $\left\{t_{1}, \ldots, t_{m}\right\} \subseteq L$. Theorem 5 is the main result of this paper.

THEOREM 5. If $\operatorname{dim} D=n$ and if $m, \omega$ are nonnegative integers such that

$$
\operatorname{dim} D\left[t_{1}, \ldots, t_{m}\right] \leqq \omega \text { for }\left\{t_{1}, \ldots, t_{m}\right\} \subseteq L,
$$

then the following conditions hold.

(1) $\operatorname{dim} D\left[X_{1}, \ldots, X_{m}\right] \leqq \omega+m$.

(2) If there exist elements $t_{1}, \ldots, t_{m}$ in $L$ such that $\operatorname{dim} D\left[t_{1}, \ldots, t_{m}\right]=\omega$, then $\operatorname{dim} D\left[X_{1}, \ldots, X_{m}\right]=\omega+m$.

Proof of (1). The proof of (1) will be by induction on $n$ and $m$. Thus, we first show that (1) is true when either $n=1$ or $m=1$.

Suppose that $n=1$. By a theorem of Sedenberg [7, p. 608], $D$ one-dimensional implies that for any $m, \operatorname{dim} D\left[X_{1}, \ldots, X_{m}\right] \leqq 2 m+1$. Clearly then (1) holds if $\omega \geqq m+1$, so we assume that $\omega \leqq m$. Since $\operatorname{dim} D\left[t_{1}, \ldots, t_{m}\right] \leqq \omega$ for $\left\{t_{1}, \ldots, t_{m}\right\} \subseteq L$, it follows, by taking $t_{\omega}=t_{\omega+1}=\cdots=t_{m}$, that $\operatorname{dim} D\left[t_{1}, \ldots, t_{\omega}\right] \leqq \omega$ for $\left\{t_{1}, \ldots, t_{\omega}\right\}$ $\subseteq L$. Theorem 1 now implies that each overring of $D$ has dimension less than or equal to $\omega$ so that, by Theorem $4 \operatorname{dim} D\left[X_{1}, \ldots, X_{m}\right] \leqq m+\omega$.

Now suppose that $m=1$. We have just seen that (1) holds for $n=1$, so we assume that (1) is true for $n<h$, that $\operatorname{dim} D=h$, and that $\operatorname{dim} D[t] \leqq \omega$ for $t \in L$. Let (0) $\subset Q_{1} \subset \ldots \subset Q_{s} \subset D[X]$ be a chain of prime ideals of $D[X]$, where $Q_{1}$ is chosen to be minimal. If $Q_{1} \cap D=(0)$, then $D[X] / Q_{1} \stackrel{S}{=}[\bar{X}]$, where $\bar{X}=X+Q_{1}$, and $f(\bar{X})=0$ for any $f \in Q_{1}$, so $\bar{X}$ is algebraic over $D$. It forlows from Corollary 1 that $\operatorname{dim} D[\bar{X}] \leqq \omega$ and this ifplies that $Q_{1}$ has depth less than or equal to $\omega$ in $D[X]$. Therefore, $s \leqq \omega+1$. On the other hand, if $Q_{1} \cap D \neq(0)$, then $Q_{1} \cap D \supseteq P$, where $P$ is a minimal prime ideal of $D$. By choice, $Q_{1}$ is minimal, and $Q_{1} \supseteq P[X]$. Thus, $Q_{1}=P[X]$. From Lemma 3 we have $\operatorname{dim}(D / P)[\sigma] \leqq \omega-1$ for each $\sigma$ in the quotient field of $D / P$. Further, $\operatorname{dim}(D / P)<h$, and by assumption (1) holds; that is,

$$
\operatorname{dim}(D / P)[X] \leqq \omega
$$

But $(D / P)[X] \cong D[X] / P[X]$ so that $P[X]=Q_{1}$ has depth less than or equal to $\omega$. Consequently $s \leqq \omega+1$ and it follows by induction that (1) is true for $m=1$.

From what we have just shown, we may make the following inductive assumptions.

(A) Suppose that (1) is true for any $n$ when $m<k$. 
(B) Suppose that (1) is true for $n<h$ when $m=k$.

Now let $\operatorname{dim} D=h$ and suppose that $\operatorname{dim} D\left[X_{1}, \ldots, X_{k}\right] \geqq \omega+k+1, \omega \geqq h$. We wish to establish the existence of $t_{1}, \ldots, t_{k}$ in $L$ such that $\operatorname{dim} D\left[t_{1}, \ldots, t_{k}\right]$ $\geqq \omega+1$.

If $\operatorname{dim} D[X] \geqq \omega+2$, then by the case in which $m=1$ there exists $t \in L$ such that $\operatorname{dim} D[t] \geqq \omega+1$. If we set $t=t_{1}=\cdots=t_{k}$, it follows that $\operatorname{dim} D\left[t_{1}, \ldots, t_{k}\right] \geqq \omega+1$ and we are finished.

Suppose, then, that $\operatorname{dim} D\left[X_{1}\right] \leqq \omega+1$. The assumption that

$$
\operatorname{dim} D\left[X_{1}\right]\left[X_{2}, \ldots, X_{k}\right] \geqq \omega+k+1
$$

then implies, by (A), that there exist elements $\delta_{2}, \ldots, \delta_{k}$ in $L\left(X_{1}\right)$ such that $\operatorname{dim} D\left[X_{1}\right]\left[\delta_{2}, \ldots, \delta_{k}\right] \geqq \omega+2$. Let $Q$ be the kernel of the canonical $D\left[X_{1}\right]$-homomorphism $\phi$ which maps $D\left[X_{1}\right]\left[X_{2}, \ldots, X_{k}\right]$ onto $D\left[X_{1}\right]\left[\delta_{2}, \ldots, \delta_{k}\right]$ in such a way that $\phi\left(X_{i}\right)=\delta_{i}$ for each $i$. Then $Q$ must have depth greater than or equal to $\omega+2$, and by Lemma $1, Q$ has height $k-1$. Hence, there exists a chain of prime ideals of $D\left[X_{1}, \ldots, X_{k}\right]$, of the form

$$
\text { (0) } \subset Q_{1} \subset \ldots \subset Q_{k-2} \subset Q \subset Q_{k} \subset \ldots \subset Q_{k+\omega+1} \subset D\left[X_{1}, \ldots, X_{k}\right] .
$$

If $f\left(X_{1}\right) \in D\left[X_{1}\right]$, then $\phi\left(f\left(X_{1}\right)\right)=f\left(X_{1}\right)$. Therefore, $Q \cap D\left[X_{1}\right]=(0)$. However, since $h(Q)=k-1$, Lemma 4 implies that $Q_{k} \cap D\left[X_{i}\right] \neq(0)$ for $i, 1 \leqq i \leqq k$. We now consider the two cases in which $Q_{k} \cap D=(0)$ and $Q_{k} \cap D \neq(0)$.

If $Q_{k} \cap D=(0)$, then $D\left[X_{1}, \ldots, X_{k}\right] / Q_{k} \cong D\left[\bar{X}_{1}, \ldots, \bar{X}_{k}\right]$, where $\bar{X}_{i}=X_{i}+Q_{k}$, and since $Q_{k} \cap D\left[X_{i}\right] \neq(0), \bar{X}_{i}$ is algebraic over $D$ for each $i$. But

$$
\operatorname{dim} D\left[\bar{X}_{1}, \ldots, \bar{X}_{k}\right] \geqq \omega+1,
$$

so by Corollary 1 there exist elements $t_{1}, \ldots, t_{k}$ in $L$ such that $\operatorname{dim} D\left[t_{1}, \ldots, t_{k}\right]$ $\geqq \omega+1$ and we are finished.

Thus, suppose that $Q_{k} \cap D \neq(0)$-say $Q_{k} \cap D=P$, where $P$ is a prime ideal of $D$ such that $h(P)=\mu$. Then $Q_{k} \supseteq P\left[X_{1}, \ldots, X_{k}\right]$ and there exists a chain $(0) \subset P_{1} \subset \ldots$ $\subset P_{\mu-1} \subset P$ of prime ideals of $D$. Let $\lambda$ be the maximal length of a proper chain of prime ideals of $D\left[X_{1}, \ldots, X_{k}\right]$ contained properly between $Q_{k}$ and $P\left[X_{1}, \ldots, X_{k}\right]$ (let $\lambda=-1$ if $Q_{k}=P\left[X_{1}, \ldots, X_{k}\right]$ ). We proceed now to show the existence of a chain of prime ideals of $D\left[X_{1}, \ldots, X_{k}\right]$ which has length greater than or equal to $k+\omega+1$ and which is of the form $Q_{k+\omega+1} \supset \cdots \supset Q_{k} \supseteq \cdots \supseteq P\left[X_{1}, \ldots, X_{k}\right]$ $\supset Q_{\gamma}^{\prime} \supset \ldots \supset Q_{1}^{\prime} \supset(0)$ where either $Q_{1}^{\prime}=P^{\prime}\left[X_{1}, \ldots, X_{k}\right]$ for some prime ideal $P^{\prime}$ of $D$, or $Q_{1}^{\prime} \cap D=(0)$ but $Q_{1}^{\prime} \cap D\left[X_{1}\right] \neq(0)$. (Here we understand that a proper chain of length $\lambda$ is contained between $Q_{k}$ and $P\left[X_{1}, \ldots, X_{k}\right]$.) We shall say that such a chain has form $(C)$. Thus, we seek a chain of form $(C)$ for which $\gamma+\lambda+\omega+3$ $\geqq k+\omega+1$.

If $\mu+\lambda \geqq k-1$, then

$$
\begin{aligned}
Q_{k+\omega+1} & \supset \ldots \supset Q_{k} \supseteq \ldots \supseteq P\left[X_{1}, \ldots, X_{k}\right] \supset P_{\mu-1}\left[X_{1}, \ldots, X_{k}\right] \\
& \supset \ldots \supset P_{1}\left[X_{1}, \ldots, X_{k}\right] \supset(0)
\end{aligned}
$$


(where a proper chain of length $\lambda$ is included between $Q_{k}$ and $P\left[X_{1}, \ldots, X_{k}\right]$ ) is such a chain since $(\mu-1)+\lambda+\omega+3=\mu+\lambda+\omega+2 \geqq k-1+\omega+2=k+\omega+1$.

Suppose, then, that $\mu+\lambda<k-1$ and consider the domain $D_{P}$. For $1 \leqq i \leqq k$, let $Q_{i}^{e}=Q_{i} D_{P}\left[X_{1}, \ldots, X_{k}\right]$, and for $1 \leqq i \leqq \mu$, let $P_{i}^{e}=P_{i} D_{P}$, where we set $Q_{k-1}=Q$ and $P_{\mu}=P$. Then $(0) \subset Q_{1}^{e} \subset \ldots \subset Q_{k}^{e}$ is a chain of prime ideals of $D_{P}, Q_{i}^{e} \cap D_{P}=(0)$ for $1 \leqq i \leqq k-1, Q_{k}^{e} \supseteq\left(P^{e}\right)\left[X_{1}, \ldots, X_{k}\right], Q_{k}^{e} \cap D_{P}=P^{e}$, and $\lambda$ is the maximal length of a chain of prime ideals of $D_{P}\left[X_{1}, \ldots, X_{k}\right]$ contained properly between $Q_{k}^{e}$ and $P^{e}\left[X_{1}, \ldots, X_{k}\right]$. By Lemma 4 there is a chain of prime ideals $P^{e}\left[X_{1}, \ldots, X_{k}\right]=$ $\Gamma_{k+1} \subset \Gamma_{k} \subset \ldots \subset \Gamma_{1} \subset D_{P}\left[X_{1}, \ldots, X_{k}\right]$ such that $\Gamma_{i} \cap D_{P}=P^{e}$ for each $i$ and such that $Q_{k}^{e}=\Gamma_{s}$ for some $s, 1 \leqq s \leqq k+1$. From Lemma 4 , and by choice of $\lambda$, it follows that $\lambda=k-s$; that is, $s+\lambda=k$. We now have the chain $(0) \subset Q_{1} \subset \ldots$ $\subset Q_{k-1} \subset \Gamma_{s} \subset \ldots \subset \Gamma_{1} \subset D_{P}\left[X_{1}, \ldots, X_{k}\right]$ of prime ideals of $D_{P}\left[X_{1}, \ldots, X_{k}\right]$, from which it follows that $\operatorname{dim} D_{P}\left[X_{1}, \ldots, X_{k}\right] \geqq s+k-1>s+\lambda+\mu=k+\mu$. Let $\gamma$ be chosen so that $s+k-1=k+\gamma+1$. Then $\gamma \geqq \mu=\operatorname{dim} D_{P}$ and, by choice of $s$, we have $s \leqq k+1$, so that $k+\gamma+1=s+k-1 \leqq 2 k$. Consequently, we have $\mu \leqq \gamma \leqq k-1$ and $\operatorname{dim} D_{P}\left[X_{1}, \ldots, X_{k}\right] \geqq k+\gamma+1$. Therefore, by Lemma 5 , there exists a chain of prime ideals of $D_{P}\left[X_{1}, \ldots, X_{k}\right]$ of the form $P^{e}\left[X_{1}, \ldots, X_{k}\right] \supset Q_{\gamma}^{\prime \prime} \supset \ldots \supset Q_{1}^{\prime \prime} \supset(0)$, where either $Q_{1}^{\prime \prime}=P^{\prime \prime}\left[X_{1}, \ldots, X_{k}\right]$ for some prime ideal $P^{\prime \prime}$ of $D_{P}$, or $Q_{1}^{\prime \prime} \cap D_{P}$ $=(0)$ but $Q_{1}^{\prime \prime} \cap D_{P}\left[X_{1}\right] \neq(0)$. Let $Q_{i}^{\prime}=Q_{i}^{\prime \prime} \cap D\left[X_{1}, \ldots, X_{k}\right]$ for each $i, 1 \leqq$ $i \leqq \gamma$. Then $Q_{\omega+k+1} \supset \ldots \supset Q_{k} \supseteq \cdots \supseteq P\left[X_{1}, \ldots, X_{k}\right] \supset Q_{\gamma}^{\prime} \supset \ldots \supset Q_{1}^{\prime} \supset(0)$ is a chain of prime ideals of $D\left[X_{1}, \ldots, X_{k}\right]$ having form $(C)$, for if $Q_{1}^{\prime \prime}=P^{\prime \prime}\left[X_{1}\right.$, $\left.\ldots, X_{k}\right]$ for some prime ideal $P^{\prime \prime}$ of $D_{P}$, then $Q_{1}^{\prime}=P^{\prime}\left[X_{1}, \ldots, X_{k}\right]$, where $P^{\prime}=P^{\prime \prime}$ $\cap D$. On the other hand, if $Q_{1}^{\prime \prime} \cap D_{P}=(0)$ but $Q_{1}^{\prime \prime} \cap D_{P}\left[X_{1}\right] \neq(0)$, then $Q_{1}^{\prime} \cap D=$ (0) but $Q_{1}^{\prime} \cap D\left[X_{1}\right] \neq(0)$. Further, $s+k-1=k+\gamma+1=\lambda+s+\gamma+1$ so that $k-1=\lambda+\gamma+1$. It then follows that $k+\omega+1=\lambda+\gamma+\omega+3$.

Lemma 6. Suppose that $\operatorname{dim} D=h$ and $\operatorname{dim} D\left[t_{1}, \ldots, t_{k}\right] \leqq \omega$ for $\left\{t_{1}, \ldots, t_{k}\right\} \subseteq L$. Then if $P$ is a proper prime ideal of $D, P\left[X_{1}, \ldots, X_{k}\right]$ has depth less than or equal to $\omega+k-1$ in $D\left[X_{1}, \ldots, X_{k}\right]$, and if $Q$ is a prime ideal of $D\left[X_{1}, \ldots, X_{k}\right]$ such that $Q \cap D=(0)$ but $Q \cap D\left[X_{1}\right] \neq(0)$, then $Q$ has depth less than or equal to $\omega+k-1$ in $D\left[X_{1}, \ldots, X_{k}\right]$.

Proof. If $P$ is a proper prime ideal of $D$, then by Lemma 3 we have

$$
\operatorname{dim}(D / P)\left[s_{1}, \ldots, s_{k}\right] \leqq \omega-h(P)
$$

for any set of elements $\left\{s_{1}, \ldots, s_{k}\right\}$ contained in the quotient field of $D / P$. From assumption (B) it then follows that $\operatorname{dim}(D / P)\left[X_{1}, \ldots, X_{k}\right] \leqq \omega+k-h(P)$. But $(D / P)\left[X_{1}, \ldots, X_{k}\right] \cong D\left[X_{1}, \ldots, X_{k}\right] / P\left[X_{1}, \ldots, X_{k}\right]$, so that $P\left[X_{1}, \ldots, X_{k}\right]$ has depth less than or equal to $\omega+k-h(P)$.

Suppose that $Q$ is a prime ideal of $D\left[X_{1}, \ldots, X_{k}\right]$ such that $Q \cap D=(0)$ but $Q \cap D\left[X_{1}\right] \neq(0)$ - say $Q \cap D\left[X_{1}\right]=Q^{\prime}$. Then $Q \supseteq\left(Q^{\prime}\right)\left[X_{2}, \ldots, X_{k}\right]$ and

$$
D\left[X_{1}, \ldots, X_{k}\right] /\left(Q^{\prime}\right)\left[X_{2}, \ldots, X_{k}\right] \cong\left(D\left[X_{1}\right] / Q^{\prime}\right)\left[X_{2}, \ldots, X_{k}\right] .
$$


But $D\left[X_{1}\right] / Q^{\prime} \cong D\left[\bar{X}_{1}\right]$, where $\bar{X}_{1}=X_{1}+Q^{\prime}$, and $\bar{X}_{1}$ is algebraic over $D$. Since $\operatorname{dim} D[t] \leqq \omega$ for $t \in L$, it follows from Corollary 1 that $\operatorname{dim} D\left[\bar{X}_{1}\right] \leqq \omega$. Moreover, by Lemma 2 , there exists a nonzero element $d$ in $D$ such that $\bar{X}_{1}$ is integral over $D[1 / d]$. But $L$ is the quotient field of $D[1 / d]$ and $\operatorname{dim} D[1 / d]\left[t_{1}, \ldots, t_{k-1}\right] \leqq \omega$ for $\left\{t_{1}, \ldots, t_{k-1}\right\} \subseteq L$. Therefore, by Theorem 3 , we have $\operatorname{dim} D\left[\bar{X}_{1}\right]\left[s_{1}, \ldots, s_{k-1}\right] \leqq \omega$ for any set of elements $\left\{s_{1}, \ldots, s_{k-1}\right\}$ of the quotient field of $D\left[\bar{X}_{1}\right]$. It now follows from assumption (A) that $\operatorname{dim} D\left[\bar{X}_{1}\right]\left[X_{2}, \ldots, X_{k}\right] \leqq \omega+k-1$. Consequently, $Q^{\prime}\left[X_{2}, \ldots, X_{k}\right]$ must have depth less than or equal to $\omega+k-1$ so that $Q$ also has depth less than or equal to $\omega+k-1$ as we wished to show.

We now complete the proof of Theorem 5 .

By assumption the ideal $Q_{1}^{\prime}$ in a chain having form $(C)$ has depth greater than or equal to $k+\omega$. However, $Q_{1}^{\prime}$ has one of the forms described in Lemma 6 so it follows that $\operatorname{dim} D\left[t_{1}, \ldots, t_{k}\right] \geqq \omega+1$ for some set $\left\{t_{1}, \ldots, t_{k}\right\} \subseteq L$.

Statement (1) of Theorem 5 now follows by induction.

Assume now that $\operatorname{dim} D\left[t_{1}, \ldots, t_{m}\right]=\omega$ for some $\left\{t_{1}, \ldots, t_{m}\right\} \subseteq L$. From (1) it follows that $\operatorname{dim} D\left[X_{1}, \ldots, X_{m}\right] \leqq \omega+m$. But if $\operatorname{dim} D\left[X_{1}, \ldots, X_{m}\right]=\alpha+m$, where $\alpha \leqq \omega$, it follows from Theorem 2 that $\operatorname{dim} D\left[s_{1}, \ldots, s_{m}\right] \leqq \alpha$ for $\left\{s_{1}, \ldots, s_{m}\right\} \subseteq L$. In particular, $\operatorname{dim} D\left[t_{1}, \ldots, t_{m}\right]=\omega \leqq \alpha$, so that $\alpha=\omega$. Statement (2) of Theorem 5 now follows.

This completes the proof of Theorem 5 .

In [3] Jaffard defines the valuative dimension, denoted by $\operatorname{dim}_{v} D$, of the domain $D$ to be the maximal rank of the valuation overrings of $D$. With this notation and terminology, we now relate many of the results of this paper in the following theorem.

THEOREM 6. Let $D$ be a finite-dimensional integral domain with identity having quotient field $L$, and let $\omega$ be a positive integer such that $\omega \geqq \operatorname{dim} D$. Then the following statements are equivalent.

(1) $\operatorname{dim}_{v} D=\omega$.

(2) Each overring of $D$ has dimension less than or equal to $\omega$ and $\omega$ is minimal.

(3) For any nonnegative integer $m, \operatorname{dim} D\left[t_{1}, \ldots, t_{m}\right] \leqq \omega$ for $\left\{t_{1}, \ldots, t_{m}\right\} \subseteq L$, and for $m \geqq \omega-1$ there exists $\left\{t_{1}, \ldots, t_{m}\right\} \subseteq L$ such that $\operatorname{dim} D\left[t_{1}, \ldots, t_{m}\right]=\omega$.

(4) For any nonnegative integer $m, \operatorname{dim} D\left[X_{1}, \ldots, X_{m}\right] \leqq m+\omega$ and for $m \geqq \omega-1$ equality holds.

(5) $\operatorname{dim} D\left[X_{1}, \ldots, X_{\omega}\right]=2 \omega$.

(6) $\operatorname{dim} D\left[t_{1}, \ldots, t_{\omega}\right] \leqq \omega$ for any set $\left\{t_{1}, \ldots, t_{\omega}\right\} \subseteq L$, and there exists a set $\left\{s_{1}, \ldots, s_{\omega}\right\} \subseteq L$ such that $\operatorname{dim} D\left[s_{1}, \ldots, s_{\omega}\right]=\omega$.

Proof. It was shown in the proof of Theorem 1 that if $D^{\prime}$ is an overring of $D$ such that $\operatorname{dim} D^{\prime}=k$, then there exists a valuation overring $V$ of $D$ such that $\operatorname{dim} V \geqq k$. This fact together with Theorem 1 shows that (1) and (2) are equivalent.

To show that (2) implies (3), it clearly suffices to show that for any positive integer $m \geqq \omega-1$, there exists $\left\{t_{1}, \ldots, t_{m}\right\} \subseteq L$ such that $\operatorname{dim} D\left[t_{1}, \ldots, t_{m}\right]=\omega$. 
However, it follows from the proof of Theorem 1 that if there exists an overring $D^{\prime}$ of $D$ such that $\operatorname{dim} D^{\prime}=\omega$, then $\operatorname{dim} D\left[t_{1}, \ldots, t_{\omega-1}\right] \geqq \omega$ for some $\left\{t_{1}, \ldots, t_{m-1}\right\}$ $\subseteq L$. Thus, equality holds and for any $m \geqq \omega-1, \operatorname{dim} D\left[t_{1}, \ldots, t_{m}\right]=\omega$, where $t_{\omega-1}=t_{\omega}=\cdots=t_{m}$.

That (3) implies (4) is an immediate consequence of Theorem 5 and certainly (4) implies (5). If (5) holds, then by Theorem 2 we have $\operatorname{dim} D\left[t_{1}, \ldots, t_{\omega}\right] \leqq \omega$ for $\left\{t_{1}, \ldots, t_{\omega}\right\} \subseteq L$. But if $\operatorname{dim} D\left[t_{1}, \ldots, t_{\omega}\right] \leqq k$ for any $k \leqq \omega$, then it follows from Theorem 5 that $\operatorname{dim} D\left[X_{1}, \ldots, X_{\omega}\right] \leqq k+\omega$ so that $k \geqq \omega$. Thus $k=\omega$, and it follows that $\operatorname{dim} D\left[s_{1}, \ldots, s_{\omega}\right]=\omega$ for some $\left\{s_{1}, \ldots, s_{\omega}\right\} \subseteq L$. Therefore (6) holds.

It is immediate from Theorem 1 that (6) implies (2) and Theorem 6 is proved.

REMARK 2. If we take $\omega=\operatorname{dim} D$, then for any nonnegative integer $m$ and $\left\{t_{1}, \ldots, t_{m}\right\} \subseteq L$, we have $\operatorname{dim} D\left[t_{1}, \ldots, t_{m}\right]=\omega$. Thus from Theorem 6 ,

$$
\operatorname{dim} D\left[X_{1}, \ldots, X_{m}\right]=m+\operatorname{dim} D
$$

for all $m$ if and only if $\operatorname{dim} D=\operatorname{dim}_{v} D$.

II. Suppose now that $D$ is integrally closed. Let $\left\{V_{\alpha}\right\}$ be the set of all valuation overrings of $D$, and let $A$ be an ideal of $D$. Then $\tilde{A}=\bigcap_{\alpha} A V_{\alpha}$ is an ideal of $D$ called the completion of $A$. If $X$ is an indeterminate over $D$ and $f \in D[X]$, then we denote by $A_{f}$ the ideal of $D$ generated by the coefficients of $f$. We now define the Kronecker function ring of $D$ as follows:

$$
D^{k}=\left\{f / g \mid f, g \in D[X], \tilde{A}_{f} \subseteq \tilde{A}_{g}\right\} .
$$

In [4], Krull shows that $D^{k}$ is an integral domain having quotient field $L(X)$ and that $D^{k} \cap L=D$. He further showed that $D^{k}$ is a Bezout domain, where a Bezout domain is defined to be a domain in which each finitely generated ideal is principal.

Now let $V$ be a valuation overring of $D$ and let $v$ be a valuation associated with $V$. If $f \in L[X]-\{0\}, f=f_{0}+f_{1} X+\cdots+f_{n} X^{n}$, we define $v^{*}(f)=\min _{0 \leqq i \leqq n}\left\{v\left(f_{i}\right) \mid f_{i} \neq 0\right\}$. Then $v^{*}$ defines a valuation on $L(X)$ having the same value group as $v$. In particular, $v$ and $v^{*}$ have the same rank. We call $v^{*}$ the trivial extension of $v$ to $L(X)$, and if $V^{*}$ is the valuation ring of $L(X)$ associated with $v^{*}$, then $V^{*}$ is called the trivial extension of $V$ to $L(X)$. Krull has shown in $\left[4\right.$, p. 560] that if $\left\{V_{\alpha}\right\}$ is the collection of valuation overrings of $D$, then $\left\{V_{\alpha}^{*}\right\}$ is the collection of valuation overrings of $D^{k}$.

An integral domain $R$ with identity is said to be a Prüfer domain provided each finitely generated nonzero ideal of $R$ is invertible. In particular, a Bezout domain if a Prüfer domain, so $D^{K}$ is a Prüfer domain. Therefore, $\operatorname{dim}_{v} D^{K}=\operatorname{dim} D^{K}[3$, p. 56]. But from the previous remarks we see that $\operatorname{dim}_{v} D=\operatorname{dim}_{v} D^{K}$. We have thus proved the following result.

THEOREM 7. Let $D$ be an integrally closed domain with identity and let $D^{K}$ be the Kronecker function ring of $D$. Then $\operatorname{dim}_{v} D=\operatorname{dim} D^{K}$.

COROLlaRY 2. If $D$ is an integral domain with identity having integral closure $\bar{D}$, the statement that $\operatorname{dim}(\bar{D})^{K}=\omega$ is equivalent to each of the statements (1)-(6) of Theorem 6. 
III. Let $D$ be an $n$-dimensional integral domain with identity having quotient field $L$. We have seen that each overring of $D$ has dimension less than or equal to $n$ if and only if $\operatorname{dim} D\left[t_{1}, \ldots, t_{n}\right] \leqq n$ for each subset $\left\{t_{1}, \ldots, t_{n}\right\} \subseteq L$. For any positive integer $n$, we now show the existence of an integral domain $D$ such that $\operatorname{dim} D=n$, and such that $\operatorname{dim} D\left[t_{1}, \ldots, t_{m}\right] \leqq n$ for any positive integer $m<n$ and for each subset $\left\{t_{1}, \ldots, t_{m}\right\} \subseteq L$, but such that $\operatorname{dim} V=n+1$ for some valuation overring $V$ of $D\left({ }^{2}\right)$. We first state the following results which are proved in [2].

LEMMA 7. Let $\left\{V_{1}, \ldots, V_{k}\right\}$ be a collection of valuation rings having quotient field $L$, and suppose that $V_{i} \ddagger V_{j}$ for $i \neq j$. If $M_{i}$ is the maximal ideal of $V_{i}$, then $\bigcap_{j \neq 1} M_{j}$ $\$ V_{i}$ for any $i$.

LEMMA 8. Let $\left\{V_{1}, \ldots, V_{k}\right\}$ be as in Lemma 7 and suppose that each $V_{i}$ contains some fixed field $F$. If $D=F+M$, where $M=M_{1} \cap \cdots \cap M_{k}$, then $D$ is a quasi-local domain with maximal ideal $M$ and if $P$ is a nonmaximal prime ideal of $D$, then $P=Q \cap D$, where $Q$ is a nonmaximal prime ideal of $V_{i}$ for some $i, 1 \leqq i \leqq k$.

Now let $n$ be an arbitrary positive integer, let $K$ be a field, and let $L=K\left(X_{1}, \ldots, X_{n+1}\right)$. We may construct valuation rings $V_{1}$ and $V_{2}$ on $L$ such that:

(a) $V_{1}$ has rank one and $V_{1}=K\left(X_{1}, \ldots, X_{n}\right)+M_{1}$, where $M_{1}$ is the maximal ideal of $V_{1}$, and $X_{n+1} \in M_{1}$.

(b) $V_{2}$ has rank $n, V_{2}=K+M_{2}$, where $M_{2}$ is the maximal ideal of $V_{2}, X_{1} / X_{n+1}$ $\in M_{2}$, and if $M_{2}=P_{1} \supset P_{2} \supset \cdots \supset P_{n} \supset P_{n+1}=(0)$ is the chain of prime ideals of $V_{2}$, then $X_{i} \in P_{i}-P_{i+1}$ for each $i, 1 \leqq i \leqq n$.

We have $X_{1} / X_{n+1} \in V_{2}-V_{1}$ and $1 / X_{1} \in V_{1}-V_{2}$. Thus, by Lemma 8, if $D=K+M$, where $M=M_{1} \cap M_{2}$, then $D$ is a quasi-local domain with maximal ideal $M$, and $D$ has quotient field $L$ since $M$ does. Further, $X_{i} X_{n+1} \in\left(P_{i} \cap D\right)-\left(P_{i+1} \cap D\right)$ for each $i, 1 \leqq i \leqq n$, so it follows from Lemma 8 that $\operatorname{dim} D=n$.

Suppose that $V$ is a nontrivial valuation overring of $D$. Then $V \supseteq M_{1} \cap M_{2}$. so by Lemma 7 either $V \subseteq V_{i}$ or $V \supseteq V_{i}$ for $i=1$ or 2 . If $V \supseteq V_{1}$, then $V=V_{1}$ since $\operatorname{dim} V_{1}=1$. If $V \subseteq V_{2}$, then $V \supseteq M_{2}$, and $V \supseteq K$ since $V \supseteq D$. Therefore, $V \supseteq K+M_{2}$ $=V_{2}$, so that equality holds. Thus, if $V$ is a nontrivial valuation overring of $D$, either $V \subseteq V_{1}$ or $V \supseteq V_{2}$.

Let $m$ be a positive integer, $m<n$, and let $\left\{t_{1}, \ldots, t_{m}\right\} \subseteq L$. Then $D\left[t_{1}, \ldots, t_{m}\right]$ is the homomorphic image of $D\left[Y_{1}, \ldots, Y_{m}\right], Y_{1}, \ldots, Y_{m}$ indeterminates over $D$, so it follows from Lemma 4 that if $P_{1} \subset P_{2} \subset \ldots \subset P_{s}$ is a chain of prime ideals of $D\left[t_{1}, \ldots, t_{m}\right]$ such that $P_{i} \cap D=M$ for each $i$, then $s \leqq m+1 \leqq n$. Further, let $D^{\prime}$ be an overring of $D$ such that $\operatorname{dim} D^{\prime} \geqq n+1$, and let $(0) \subset P_{1}^{\prime} \subset \ldots \subset P_{n+1}^{\prime} \subset D^{\prime}$ be a chain of prime ideals of $D^{\prime}$. Then there exists a valuation overring $V$ of $D^{\prime}$, and a chain $(0) \subset Q_{1} \subset \ldots \subset Q_{n+1} \subset V$ of prime ideals of $V$ such that $Q_{i} \cap D^{\prime}=P_{i}^{\prime}$, $1 \leqq i \leqq n+1\left[5\right.$, p. 37]. Since $\operatorname{dim} V \geqq n+1, V \ddagger V_{2}$. Therefore, $V \subseteq V_{1}$, so it follows

$\left.{ }^{2}\right)$ The method for constructing such an example was suggested by William Heinzer. 
that $M_{1} \subseteq Q_{1}$. Thus $P_{1}^{\prime} \cap D=\left(Q_{1} \cap D^{\prime}\right) \cap D \supseteq\left(M_{1} \cap D^{\prime}\right) \cap D=M$, and consequently, $P_{i}^{\prime} \cap D=M$ for each $i, 1 \leqq i \leqq n+1$. From what we have shown it follows that $\operatorname{dim} D\left[t_{1}, \ldots, t_{m}\right] \leqq n$ for $\left\{t_{1}, \ldots, t_{m}\right\} \subseteq L$. But we may construct a valuation ring $V_{3}$ on $L$ such that $V_{3}$ has rank $n+1$ and $V_{3}=K+M_{3}$, where $M_{3}$ is the maximal ideal of $V_{3}$ and $M_{3} \supseteq M_{1}$. Then $V_{3} \supseteq K+M_{1} \supseteq D$.

\section{REFERENCES}

1. R. W. Gilmer, Jr., Domains in which valuation ideals are prime powers, Arch. Math. 17 (1966), 210-215.

2. R. W. Gilmer, Jr. and William Heinzer, Primary ideals and valuation ideals. II, Trans. Amer. Math. Soc. 131 (1968), 149-162.

3. P. Jaffard, Théorie de la dimension dans les anneaux de polynomes, Gauthier-Villars, Paris, 1960.

4. W. Krull, Beitrage zur Arithmetik kommutativer Integritätsbereiche, Math. Z. 41 (1936), 545-577.

5. M. Nagata, Local rings, Interscience, New York, 1962.

6. A. Seidenberg, A note on the dimension theory of rings, Pacific J. Math. 3 (1953), 505-512.

7. - A note on the dimension theory of rings. II, Pacific J. Math. 4 (1954), 603-614.

8. O. Zariski and P. Samuel, Commutative algebra, Vol. 1, Van Nostrand, Princeton, N. J., 1958.

9. - Commutative algebra, Vol. 2, Van Nostrand, Princeton, N. J., 1960.

Florida State University,

TAllahassee, Florida 